\title{
Research on Taxi Driver Strategy Game Evolution with Carpooling Detour
}

\author{
Wei Zhang, Ruichun He $\mathbb{D}^{\text {, }}$, Changxi Ma $\mathbb{D}^{\mathbb{D}}$, and Mingxia Gao \\ School of Traffic and Transportation, Lanzhou Jiaotong University, Lanzhou 730070, China \\ Correspondence should be addressed to Ruichun He; tranman@163.com
}

Received 9 October 2017; Accepted 31 December 2017; Published 28 January 2018

Academic Editor: Giulio E. Cantarella

Copyright (C) 2018 Wei Zhang et al. This is an open access article distributed under the Creative Commons Attribution License, which permits unrestricted use, distribution, and reproduction in any medium, provided the original work is properly cited.

\begin{abstract}
For the problem of taxi carpooling detour, this paper studies driver strategy choice with carpooling detour. The model of taxi driver strategy evolution with carpooling detour is built based on prospect theory and evolution game theory. Driver stable strategies are analyzed under the conditions of complaint mechanism and absence of mechanism, respectively. The results show that passenger's complaint mechanism can effectively decrease the phenomenon of driver refusing passengers with carpooling detour. When probability of passenger complaint reaches a certain level, the stable strategy of driver is to take carpooling detour passengers. Meanwhile, limiting detour distance and easing traffic congestion can decrease the possibility of refusing passengers. These conclusions have a certain guiding significance to formulating taxi policy.
\end{abstract}

\section{Introduction}

Taxi carpooling mode has the characteristics of improving the transportation efficiency, easing the traffic pressure, and reducing environmental pollution $[1,2]$. In recent years many cities in China have begun to implement taxi carpooling policy to solve the serious traffic problems. Taxi carpooling operation is in the initial stage, and the carpool policy is imperfect. It is very necessary to study taxi carpooling problem in the present situation.

Many scholars have carried out researches on the problem of carpooling. At present, most researches on carpooling are focused on the problem of carpooling matching and path optimization [3-6]. Aissat and Oulamara [7] proposed a method of optimizing starting point position of driver and passenger based on the heuristic algorithm, and the results can minimize the travel cost and detour distance. Santi et al. [8] proposed a method of carpooling strategy optimization based on network, which can shorten the passenger travel time and improve the utilization rate of vehicles. Shinde and Thombre [9] solved the problem of carpooling path optimization based on genetic algorithms. He et al. [10] obtained the optimal path by mining GPS positioning data. Nourinejad and Roorda [11] designed a centralized and decentralized optimization algorithms based on binary integer programming and dynamic auction-based multiagent for the problem of matching passengers and drivers. Huang et al. [12] proposed the genetic-based carpooling route and matching algorithm for the multiobjective optimization problem. Xia et al. [13] built an optimization model of carpooling matching and designed heuristic algorithm to solve it. Chiou and Chen [14] proposed a dynamic matching method for carpooling, which can be used in mobile devices via ad hoc Wi-Fi networks. Boukhater et al. [15] presented a GA with a customized fitness function that searches for the solution with minimal travel distance, efficient ride matching, timely arrival, and maximum fairness, which can be used in carpooling system. Xiao et al. [16] studied passengers matching problem based on clustering and pattern recognition methods. The above researches establish the model of carpooling matching optimization and then find the optimal matching mode by heuristic algorithm or data mining algorithms, which solve the problem of the carpooling matching and path optimization and provide theory support for carpooling implementation.

Detour problem is a common phenomenon of taxi carpooling. In general detour passengers will get more cost discount than usual carpooling to make up for the losses caused by detour. But from the perspective of the drivers, 


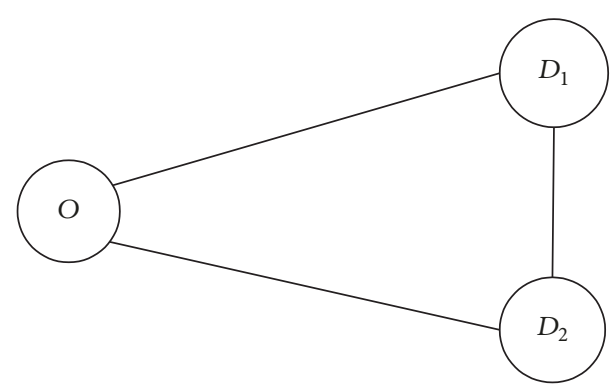

FIGURE 1: Travel routes of carpooling passengers.

drivers' income will be affected. Driver may be more resistant to carpooling detour, which makes carpooling detour impossible in reality. So it is necessary to study the problem of driver strategy with carpooling detour. The study has an important significance to the implementation of carpooling system. However few researches involve the problem of carpooling detour. Therefore this paper establishes a model of driver strategy evolution based on prospect theory and evolutionary game theory and studies the problem of driver strategy with carpooling detour.

\section{Problem Description and Research Framework}

2.1. Problem Description. Suppose two passengers depart from the same location $O$. The destination of passenger 1 is $D_{1}$ and that of passenger 2 is $D_{2}$. The best path for passenger 1 is $O D_{1}$ and passenger 2 is $O D_{2}$. $O D_{1}$ and $O D_{2}$ do not coincide, as shown in Figure 1. There are three road sections in Figure 1, where $O D_{1}$ is section $1, O D_{2}$ is section $2, D_{1} D_{2}$ is section 3 , and $\left|O D_{1}\right|=l_{1},\left|O D_{2}\right|=l_{1},\left|D_{1} D_{2}\right|=l_{3}$. The traffic status of each section is uncertain. The traffic status of the section $i$ is represented as $j_{i}$. When the traffic status of the section $i$ is normal, $j_{i}=1$; When the status is congestion, $j_{i}=2$. The traffic congestion rate of the section $i$ is represented as $\varphi_{i}$. The arrival time of the section $i$ with normal traffic is represented as $t_{i}$. The waiting time of the section $i$ with congestion is represented as $r_{i}$. Now the two passengers ride the same taxi through path $O D_{1} D_{2}$. That is, passenger 1 is sent to his destination first, and then passenger 2. Passenger 1 will pay a part of the fare, and payment ratio is $\theta$. Passenger 2 will get more discount than passenger 1 because he makes a detour. The driver faces with two strategies: taking the two passengers or refusing them. This paper will study the problem of drivers' stability strategy under the above conditions.

2.2. Research Framework. The evolution game theory is an effective method to study human stability strategy. The theory analyzes problem on the premise of the bounded rationality. It believes that the human behaviors reveal bounded rationality, and human strategies reach stability gradually through constant adjustment [17, 18]. Evolutionary game theory studies stable strategies of human behaviors, which solves the problem of full rational analysis divorcing from reality [19-21]. The evolution game theory has become one of the most important fields of modern game theory.

The evolutionary game model has been applied into analysis of stable strategies with bounded rationality, but the theory model still shows the defect, which has characteristic of full rationality. In the payoff matrix of the evolutionary game model, revenue value is usually defined as direct benefits of each actor under different strategies. That is, the fundamental condition of evolutionary game is that actor can accurately obtain revenue values of different strategies, which coincides with traditional full rational game. But the actors whose cognitive ability, computational ability, and judgment ability cannot achieve such a high requirement are not full rational. So the actors are not able to obtain accurate revenue values. Then the fundamental condition of evolutionary game can not be satisfied, and the subsequent evolutionary analysis will lose significance.

In this paper, prospect theory is introduced into evolutionary game model to solve above problem. Prospect theory is a cross research theory of psychology and behavioral science, which reveals the human psychology of decisionmaking. This theory describes people's decision psychology rules in uncertain environments [22], and reflects more truly people's behavior tendency. According to prospect theory, strategy prospect value describes people's perception results of revenue for the strategy with respect to personal psychological expectations $[23,24]$. So strategy prospect value is more close to people's judgment result, reflecting bounded rationality [25-27]. Therefore, this paper combines prospect theory and evolutionary game theory and establishes driver strategy evolution model based on prospect value to study driver stable strategy under the condition of carpooling detour, with strategic prospect value instead of strategic revenue value of game model. The model includes two parts: driver strategy prospect model and evolutionary game model. Firstly, driver strategy prospect model is established. The prospect value of each strategy is obtained through the established model. Then evolutionary game model is built based on strategy prospect value, using strategy prospect value as strategic revenue. Driver stable strategy is analyzed by replicated dynamic mechanism. This method improves the traditional evolutionary game model and makes up for the full rationality shortcoming of payoff matrix in the traditional evolutionary game model, which makes research work closer to reality.

This paper studies driver stable strategy based on the improved model. The study finds out that refusing behaviors appear easily with carpooling detour. So passenger complaint mechanism is introduced next. Driver stable strategy is studied with passenger complaint mechanism. Eventually, we confirm that passenger complaint mechanism is an effective method to avoid refusing behaviors. We also find out related regulations.

\section{Modeling}

3.1. Driver Strategy Prospect Value. Suppose taxi charging standard stipulates that initiate fee is $f_{s} ¥ / d \mathrm{~km}, f_{r} ¥$ per kilometer more than $d$ kilometers, and waiting fee is $f_{q} ¥ / \mathrm{min}$. 
Then when travel distance is $l$ and waiting time is $r$, $\operatorname{cost} Z(l, r)$ is

$$
Z(l, r)= \begin{cases}f_{s}+r f_{q} & \text { if } l \leq d \\ (l-d) f_{r}+f_{s}+r f_{q} & \text { if } l>d .\end{cases}
$$

If two passengers take the same taxi, cost of passenger 1 is

$$
C_{1}= \begin{cases}\theta Z\left(l_{1}, 0\right) & \text { if } j_{1}=1 \\ \theta Z\left(l_{1}, r_{1}\right) & \text { if } j_{1}=2 .\end{cases}
$$

The travel route causes detour for passenger 2. So it is necessary to reduce the cost of passenger 2 to make up for his time loss. Considering that the more the detour time the lower the detour cost, the cost of passenger 2 is inversely proportional to his nondetour time, following relational expression given.

$$
C_{2}= \begin{cases}\theta Z\left(l_{2}, 0\right) \frac{t_{2}}{t_{1}+t_{3}} & \text { if } j_{1}=1, j_{3}=1 \\ \theta Z\left(l_{2}, 0\right) \frac{t_{2}}{t_{1}+t_{3}+r_{3}} & \text { if } j_{1}=1, j_{3}=2 \\ \theta Z\left(l_{2}, 0\right) \frac{t_{2}}{t_{1}+t_{3}+r_{1}} & \text { if } j_{1}=2, j_{3}=1 \\ \theta Z\left(l_{2}, 0\right) \frac{t_{2}}{t_{1}+t_{3}+r_{1}+r_{3}} & \text { if } j_{1}=2, j_{3}=2 .\end{cases}
$$

If driver takes the two passengers in carpooling mode, starting from $O$, through $D_{1}$ before arriving in $D_{2}$, the driver's income is the sum of the two passengers' costs.

$$
I=C_{1}+C_{2} .
$$

If driver takes only a passenger, starting from $O$, through $D_{1}$ before arriving in $D_{2}$, the driver's income is

$$
I^{0}= \begin{cases}Z\left(l_{1}+l_{3}, 0\right) & \text { if } j_{1}=1, j_{3}=1 \\ Z\left(l_{1}+l_{3}, r_{3}\right) & \text { if } j_{1}=1, j_{3}=2 \\ Z\left(l_{1}+l_{3}, r_{1}\right) & \text { if } j_{1}=2, j_{3}=1 \\ Z\left(l_{1}+l_{3}, r_{1}+r_{3}\right) & \text { if } j_{1}=2, j_{3}=2 .\end{cases}
$$

The driver's income $I_{0}$ when he takes only a passenger which is regarded as driver reference point; then the driver's gain relative to reference point is

$$
y^{D}=I-I^{0} .
$$

According to prospect theory, value function is defined as follows:

$$
V\left(y^{D}\right)= \begin{cases}\left(y^{D}\right)^{\alpha} & \text { when } y^{D} \geq 0 \\ -\lambda\left(-y^{D}\right)^{\beta} & \text { when } y^{D}<0,\end{cases}
$$

where $\alpha$ and $\beta(0 \leq \alpha, \beta \leq 1)$ are risk attitude coefficients. The bigger $\alpha$ or $\beta$ is, the more adventurous decision maker tends to. $\lambda(\lambda>1)$ is loss aversion coefficient, which reflects that decision maker is more sensitive to loss. Value function shows the decreasing sensitivity in the two directions of gain and loss. The result of the value function reflects the psychological revenue more realistically. The parameters values $\alpha=0.89, \beta=0.92, \lambda=2.25$ are more consistent with psychological characteristic of decision maker [22].

Path $O D_{1} D_{2}$ includes section 1 and section 3. Traffic congestion rate of the path is defined as $\varphi_{13}$.

$$
\varphi_{13}= \begin{cases}\left(1-\varphi_{1}\right)\left(1-\varphi_{3}\right) & \text { if } j_{1}=1, j_{3}=1 \\ \left(1-\varphi_{1}\right) \varphi_{3} & \text { if } j_{1}=1, j_{3}=2 \\ \varphi_{1}\left(1-\varphi_{3}\right) & \text { if } j_{1}=2, j_{3}=1 \\ \varphi_{1} \varphi_{3} & \text { if } j_{1}=2, j_{3}=2 .\end{cases}
$$

The probability of traffic congestion which is perceived by passenger is different from actual probability according to prospect theory. The perceived probability of traffic congestion is

$$
W\left(\varphi_{13}\right)= \begin{cases}\frac{\left(\varphi_{13}\right)^{\chi}}{\left(\left(\varphi_{13}\right)^{\chi}+\left(1-\varphi_{13}\right)^{\chi}\right)^{1 / \chi}} & \text { if } y^{D} \geq 0 \\ \frac{\left(\varphi_{13}\right)^{\delta}}{\left(\left(\varphi_{13}\right)^{\delta}+\left(1-\varphi_{13}\right)^{\delta}\right)^{1 / \delta}} & \text { if } y^{D}<0 .\end{cases}
$$

The parameter values most reflecting individual behavior of decision maker are $\chi=0.61, \delta=0.69$ [22]. The transformation reflects that people tend to overestimate small probability events and underestimate medium and large probability events, while people are relatively insensitive to the intermediate stage. Transformed probability is more close to people's subjective probability.

If the driver selects taking carpooling passenger strategy, the prospect value $E V_{1}^{D}$ is

$$
\begin{gathered}
E V_{1}^{D}=\sum_{v=1}^{2} \sum_{u=1}^{2} W\left(\varphi_{13}\left(j_{1}=u, j_{3}=v\right)\right) V \\
\cdot\left(y^{D}\left(j_{1}=u, j_{3}=v\right)\right),
\end{gathered}
$$

where $\varphi_{13}\left(j_{1}=u, j_{3}=v\right)$ is traffic congestion probability under the condition of $j_{1}=u$ and $j_{3}=v \cdot y^{D}\left(j_{1}=u, j_{3}=v\right)$ is driver gain under the condition of $j_{1}=u$ and $j_{3}=v$.

Because driver reference point is the gain when he takes only a passenger, the prospect value is 0 if the driver takes only a passenger; the prospect value is the result of $I=0$ condition if the driver does not take any passenger.

3.2. Evolutionary Analysis of Driver Carpooling Strategy. Driver has two strategy choices: taking carpooling detour passengers (strategy 1) and refusing carpooling detour passengers (strategy 2). If the driver selects strategy 1 , and the passengers select sharing the taxi, carpooling succeeds; then the driver's prospect value is represented as $E V_{1}^{D}$; If the driver selects strategy 2 , and the passengers select riding the taxi lonely, the driver takes only a passenger; then the prospect 
value is 0 . If the driver's strategy and passengers' strategy are not different, the driver does not take any passenger and the prospect value is represented as $E V_{2}^{D}$.

Suppose the percentage of passengers choosing carpooling detour strategy is $p_{1}$, and the percentage of drivers choosing carpooling detour strategy is $q$. Expected gains of driver choosing strategy 1 and strategy 2 respectively are as follows.

$$
\begin{aligned}
& u_{1}=E V_{1}^{D} p_{1}+E V_{2}^{D}\left(1-p_{1}\right) \\
& u_{2}=E V_{2}^{D} p_{1} .
\end{aligned}
$$

Average expected gain is

$$
\begin{aligned}
\bar{u} & =u_{1} q+u_{2}(1-q) \\
& =E V_{1}^{D} p_{1} q+E V_{2}^{D}\left(1+p_{1}(1-2 q)\right) .
\end{aligned}
$$

Replicator dynamic equation of driver percentage is

$$
\begin{aligned}
F(q) & =\frac{d q}{d t}=q\left(u_{1}-\bar{u}\right) \\
& =q(1-q)\left(\left(E V_{1}^{D}-2 E V_{2}^{D}\right) p_{1}+E V_{2}^{D}\right) .
\end{aligned}
$$

Suppose $F(q)=d q / d t=0$; then $q=0, q=1, p_{1}^{*}=$ $E V_{2}^{D} /\left(2 E V_{2}^{D}-E V_{1}^{D}\right)$.

According to the condition, we know $E V_{2}^{D}<0$. The stability analysis is as follows:

(1) When $E V_{1}^{D}-2 E V_{2}^{D}>0$,

(1) if $p_{1}=p_{1}^{*}$, then $F(q)=0, F^{\prime}(q)=0$; here any strategy may be stable;

(2) if $p_{1}>p_{1}^{*}$, then $F^{\prime}(0)>0, F^{\prime}(1)<0$; here $q=1$ is stable strategy. That is, when the percentage of passengers choosing carpooling is more than $p_{1}^{*}$, driver will choose strategy 1 ;

(3) if $p_{1}<p_{1}^{*}$, then $F^{\prime}(0)<0, F^{\prime}(1)>0$; here $q=0$ is stable strategy. That is, when the percentage of passengers choosing carpooling is low than $p_{1}^{*}$, driver will choose strategy 2.

(2) When $E V_{1}^{R}-2 E V_{2}^{R}<0$, inevitably $p_{1}>p_{1}^{*}$; here $q=0$ is stable strategy, and driver will choose strategy 2 .

Through the above analysis, driver stable strategy is taking passengers only if $E V_{1}^{D}-2 E V_{2}^{D}>0$ and $p_{1}>p_{1}^{*}$; otherwise stable strategy is refusing passengers.

\section{Researches on Driver Strategy Evolution with Complaint Mechanism}

Driver income will be affected when detour occurs in carpooling. Drivers are inclined to refusing passengers. Next passenger complaint mechanism is introduced to further analyze driver strategy change with complaint mechanism.

4.1. Prospect Value of Driver Strategy with Complaint Mechanism. Under the condition of passenger complaint mechanism, driver will make a choice carefully after considering the consequences of refusing passengers. Driver has two

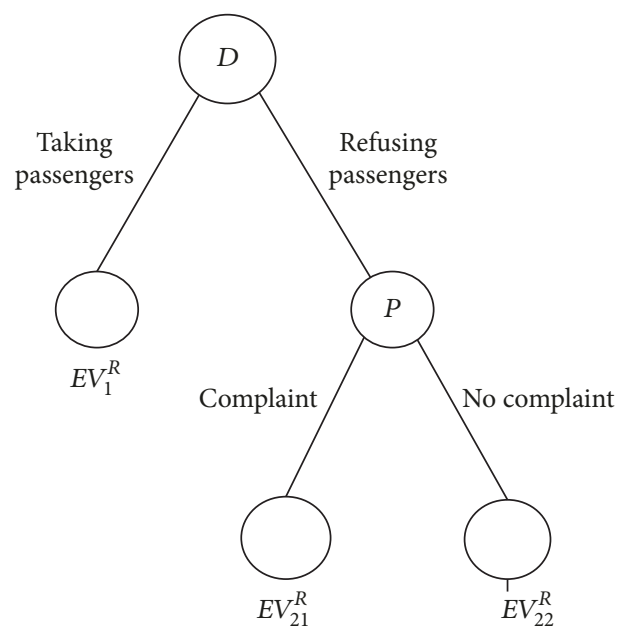

FIGURE 2: Driver strategies and prospect values.

choices: taking passengers and refusing passengers. When the behavior of refusing passengers occurs, passengers will choose the complaint strategies or no complaint strategies. The specific strategies are shown in Figure 2. If driver selects taking carpooling detour passengers, the prospect value is still $E V_{1}^{D}$; if driver selects refusing passengers, and passengers select complaint, the prospect value is $E V_{21}^{D}$; if driver selects refusing passengers, and passengers select no complaint, the prospect value is $E V_{22}^{D}$.

When the driver selects refusing strategy (assuming that only passenger 1 is taken at this time), and the passengers select complaint strategy, the prospects value of the driver is

$$
E V_{21}^{D}=\sum_{u=1}^{2} W\left(\varphi_{13}\left(j_{1}=u\right)\right) V(-g),
$$

where $g$ is punishment for refusing behavior after complaint.

When the driver selects refusing strategy, and the passengers select no complaint strategy, the prospects value of the driver is

$$
E V_{22}^{D}=0
$$

4.2. Stability Strategy Analysis. Suppose the percentage of passengers choosing complaint strategy is $p_{2}$, and the percentage of drivers choosing carpooling detour strategy is $q$. Expected gains of driver choosing strategy 1 and strategy 2, respectively, are as follows.

$$
\begin{aligned}
& u_{11}=E V_{1}^{D} \\
& u_{12}=E V_{21}^{D} p_{2}+E V_{22}^{D}\left(1-p_{2}\right) .
\end{aligned}
$$

Average expected gain is

$$
\begin{aligned}
\bar{u}_{1} & =u_{11} q+u_{12}(1-q) \\
& =E V_{1}^{D} q+\left(E V_{21}^{D} p_{2}+E V_{22}^{D}\left(1-p_{2}\right)\right)(1-q) .
\end{aligned}
$$


Replicator dynamic equation of driver percentage is

$$
\begin{aligned}
& F(q)=\frac{d q}{d t}=q\left(u_{11}-\bar{u}_{1}\right)=q\left(E V_{1}^{D}\right. \\
& \left.-\left(E V_{1}^{D} q+\left(E V_{21}^{D} p_{2}+E V_{22}^{D}\left(1-p_{2}\right)\right)(1-q)\right)\right) \\
& =q(1-q)\left(E V_{1}^{D}-E V_{21}^{D} p_{2}-E V_{22}^{D}\left(1-p_{2}\right)\right) .
\end{aligned}
$$

Suppose $F^{\prime}(q)=0$; then $q=0, q=1, p_{2}^{*}=\left(E V_{22}^{D}-\right.$ $\left.E V_{1}^{D}\right) /\left(E V_{22}^{D}-E V_{21}^{D}\right)$. Due to $E V_{22}^{D}=0, p_{2}^{*}=E V_{1}^{D} / E V_{21}^{D}$.

According to the condition, we know $E V_{21}^{D}<0$. The stability analysis is as follows:

(1) When $E V_{1}^{D}<0$,

(1) if $p_{2}=p_{2}^{*}$, then $F(q)=0, F^{\prime}(q)=0$; here any strategy may be stable.

(2) if $p_{2}>p_{2}^{*}$, then $F^{\prime}(0)>0, F^{\prime}(1)<0$; here $q=1$ is stable strategy. That is, when the percentage of passengers choosing carpooling is more than $p_{2}^{*}$, driver will choose strategy 1 .

(3) if $p_{2}<p_{2}^{*}$, then $F^{\prime}(0)<0, F^{\prime}(1)>0$; here $q=0$ is stable strategy. That is, when the percentage of passengers choosing carpooling is lower than $p_{2}^{*}$, driver will choose strategy 2.

(2) When $E V_{1}^{D}>0$, inevitably $E V_{1}^{D} / E V_{21}^{D}<0, p_{1}>p_{1}^{*}$; here $q=1$ is stable strategy, and driver will choose strategy 1 .

Through the above analysis, drivers will select taking carpooling passengers strategy unless $E V_{1}^{D}<0$ and $p_{2}>p_{2}^{*}$.

\section{Driver Evolution Strategies Comparison under the Different Mechanisms}

(1) When $E V_{1}^{D}>0$. Only to meet the condition that the initial percentage of passengers choosing carpooling detour strategy is more than $p_{1}^{*}$ is drivers' stable strategy taking carpooling detour passengers under the absence of complaint mechanism; however, drivers' stable strategy is taking carpooling detour passengers under the compliant mechanism.

(2) When $E V_{1}^{D}<0$

(1) If $E V_{1}^{D}-2 E V_{2}^{D}<0$. Drivers' stable strategy is refusing carpooling passengers under the absence of complaint mechanism; however drivers' stable strategy is taking carpooling detour passengers under the compliant mechanism as long as the condition that the initial percentage of passengers choosing complaint strategy is more than $p_{2}^{*}$ is satisfied.

(2) If $E V_{1}^{D}-2 E V_{2}^{D}>0$. If the initial percentage of passengers choosing carpooling detour strategy is more than $p_{1}^{*}$, drivers' stable strategy is taking carpooling passengers under the absence of complaint mechanism; if the initial percentage of choosing complaint strategy is more than $p_{2}^{*}$, drivers' stable strategy is taking carpooling passengers under the compliant mechanism.

Under the above conditions, prove $p_{1}^{*}>p_{2}^{*}$ as follows.
Proof.

$$
\begin{aligned}
& \frac{E V_{2}^{D}}{2 E V_{2}^{D}-E V_{1}^{D}}-\frac{E V_{1}^{D}}{E V_{21}^{D}} \\
& =\frac{E V_{2}^{D} E V_{21}^{D}-\left(2 E V_{2}^{D}-E V_{1}^{D}\right) E V_{1}^{D}}{\left(2 E V_{2}^{D}-E V_{1}^{D}\right) E V_{21}^{D}} \\
& =\frac{E V_{2}^{D}\left(E V_{21}^{D}-2 E V_{1}^{D}\right)+\left(E V_{1}^{D}\right)^{2}}{\left(2 E V_{2}^{D}-E V_{1}^{D}\right) E V_{21}^{D}} \\
& =\frac{\left(E V_{1}^{D}-E V_{2}^{D}\right)^{2}+E V_{2}^{D}\left(E V_{21}^{D}-E V_{2}^{D}\right)}{\left(2 E V_{2}^{D}-E V_{1}^{D}\right) E V_{21}^{D}} .
\end{aligned}
$$

Due to $E V_{1}^{D}-2 E V_{2}^{D}>0, E V_{21}^{D}<0, E V_{2}^{D}<0$ and $E V_{21}^{D}<$ $E V_{2}^{D}$, we can know $E V_{2}^{D} /\left(2 E V_{2}^{D}-E V_{1}^{D}\right)-E V_{1}^{D} / E V_{21}^{D}>0$, that is $p_{1}^{*}>p_{2}^{*}$.

Through the above analysis, the complaint mechanism makes the conditions of driver stabilizing at taking carpooling detour strategy easier to be satisfied. The complaint mechanism can reduce the rejection rate of drivers.

\section{Example Analysis}

Suppose taxi charging standard stipulates that initiate fee is $10 ¥$ per $3 \mathrm{~km}, 1.4 ¥$ per kilometer more than 3 kilometers, and waiting fee is $1.2 ¥$ per $2.5 \mathrm{~min}$. Two passengers set out from the same location $O$ to different destinations. The destination of passenger 1 is $D_{1}$ and that of passenger 2 is $D_{2}$. They intend to ride the same taxi. It is known that $l_{1}=l_{2}=5$ and $l_{3}=1$. Travel speed is $30 \mathrm{~km}$ per hour. Travel time is twice as normal condition when traffic congestion occurs. The traffic congestion rate of each section is $\varphi=0.5$, and carpooling payment ratio is $\theta=0.7$.

We can know $p_{1}^{*}=E V_{2}^{R} /\left(2 E V_{2}^{R}-E V_{1}^{R}\right)=0.6$ under the absence of complaint mechanism. $q=1$ is drivers' stable strategy when $p>0.6$. That is, drivers' stable strategy is taking passengers if the initial percentage of passengers choosing carpooling detour strategy is more than $60 \%$.

Suppose driver's punishment is $g=50$ under the complaint mechanism. We can know $p_{2}^{*}=\left(E V_{22}^{R}-E V_{1}^{R}\right) /\left(E V_{22}^{R}-\right.$ $\left.E V_{21}^{R}\right)=0.17$ under the condition. $q=1$ is drivers' stable strategy when $p>0.17$. That is, drivers' stable strategy is taking passengers if the initial percentage of passengers choosing complaint strategy is more than $17 \%$.

So under the complaint mechanism, the conditions that cause driver to select carpooling strategy are more easily satisfied, and drivers tend to take carpooling passengers at this time.

Thresholds $p_{1}^{*}$ and $p_{2}^{*}$ are influenced by the factors, such as detour distance, penalty, payment ratio, and traffic congestion rate. The influence analysis is as follows.

Figure 3 shows the influences of detour distance on the threshold under the two different mechanisms. Figure 3(a) is the influences analysis under the absence of complaint mechanism, and Figure 3(b) is the influences analysis under 


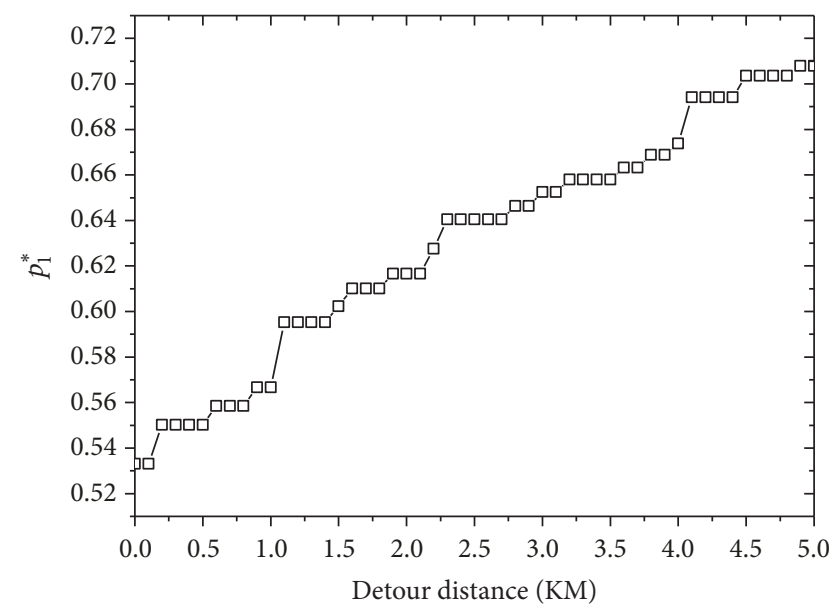

(a) Influence of detour distance on threshold under the absence of complaint mechanism

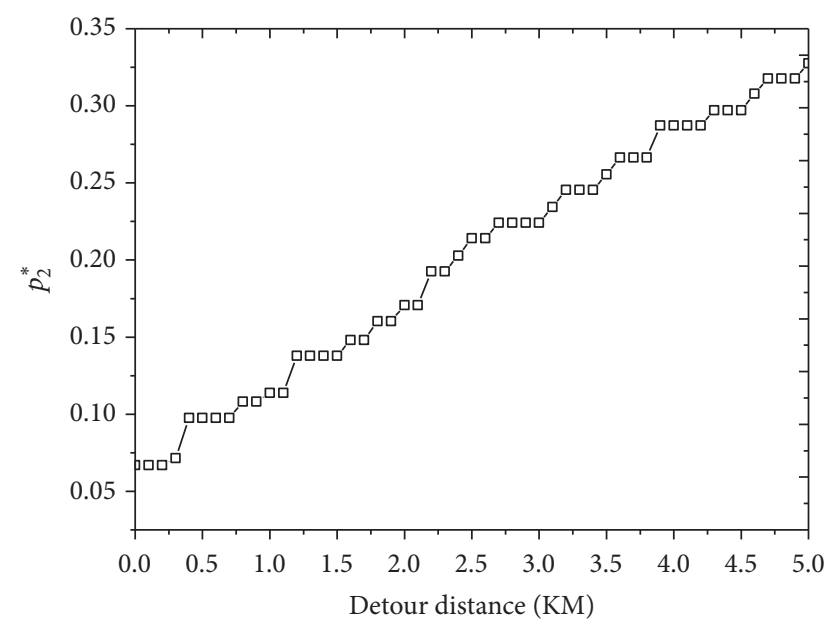

(b) Influence of detour distance on threshold under the complaint mechanism

FIGURE 3: Influence of detour distance on threshold.

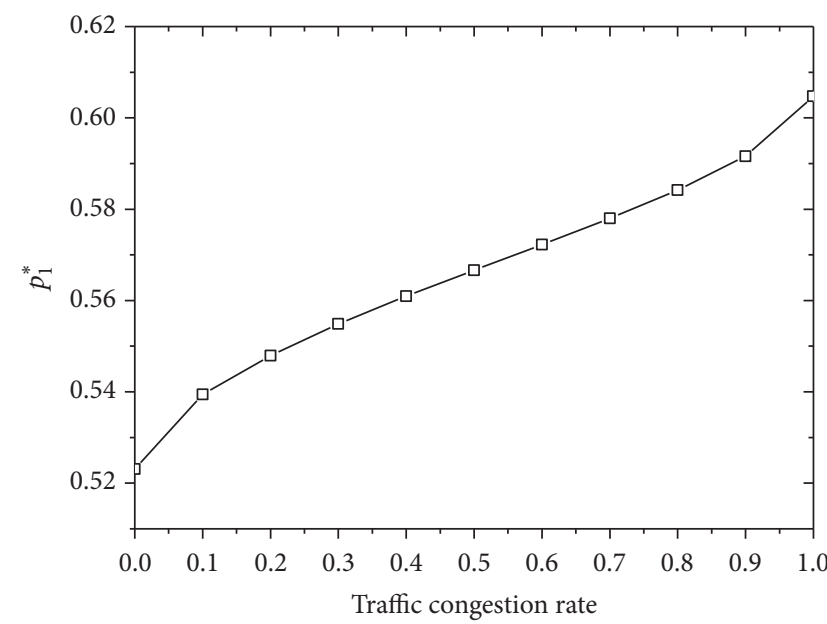

(a) Influence of traffic congestion rate on threshold under the absence of complaint mechanism

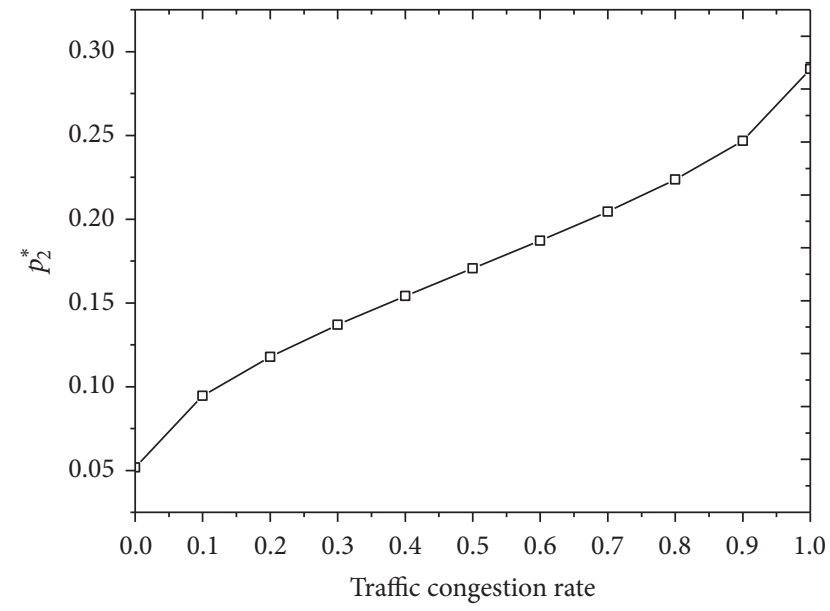

(b) Influence of traffic congestion rate on threshold under the complaint mechanism

FIGURE 4: Influence of traffic congestion rate on threshold.

the complaint mechanism. We can see that the threshold increases gradually with detour distance increases. Under the absence of complaint mechanism, the initial percentage of passengers choosing carpooling strategy needs to reach 53\% when detour distance is 0 ; the initial percentage needs to reach $71 \%$ when detour distance is $5 \mathrm{KM}$. However, under the complaint mechanism, the initial percentage of passengers choosing complaint strategy needs to only reach $7 \%$ when detour distance is 0 ; the initial percentage needs to reach $33 \%$ when detour distance is $5 \mathrm{KM}$.

Figure 4 shows the influences of traffic congestion rate on the threshold under the two different mechanisms. Figure 4(a) is the influences analysis under the absence of complaint mechanism, and Figure $4(\mathrm{~b})$ is the influences analysis under the complaint mechanism. We can see that the threshold increases gradually with traffic congestion rate increases. Under the absence of complaint mechanism, the initial percentage of passengers choosing carpooling strategy needs to reach $52 \%$ when traffic is certainly normal; the initial percentage needs to reach $60 \%$ when traffic congestion occurs inevitably. However, under the complaint mechanism, the initial percentage of passengers choosing complaint strategy needs to only reach $5 \%$ when traffic is certainly normal; the initial percentage needs to reach $28 \%$ when traffic congestion occurs inevitably.

Figure 5 shows the influences of passenger payment ratio on the threshold under the two different mechanisms. Figure 5(a) is the influences analysis under the absence of complaint mechanism, and Figure $5(\mathrm{~b})$ is the influences analysis under the complaint mechanism. We can see that the 


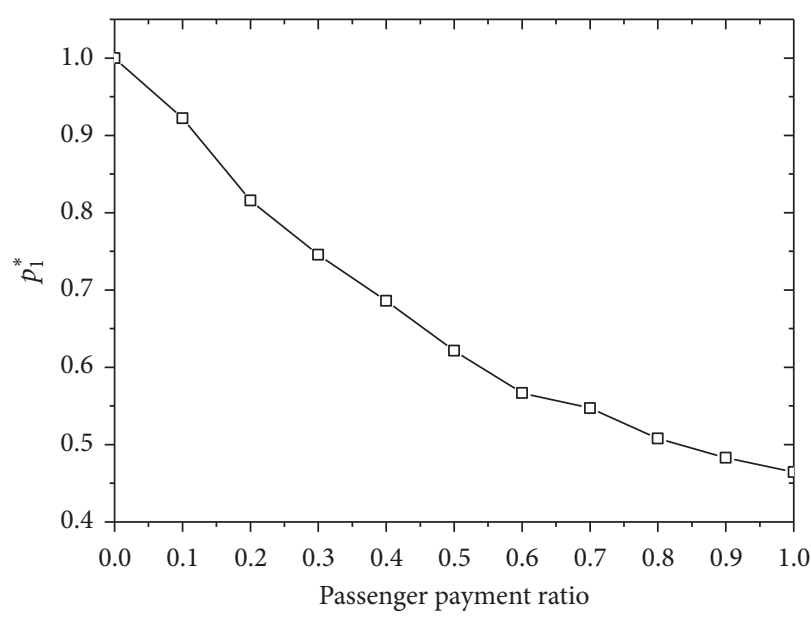

(a) Influence of payment ratio on threshold under the absence of complaint mechanism

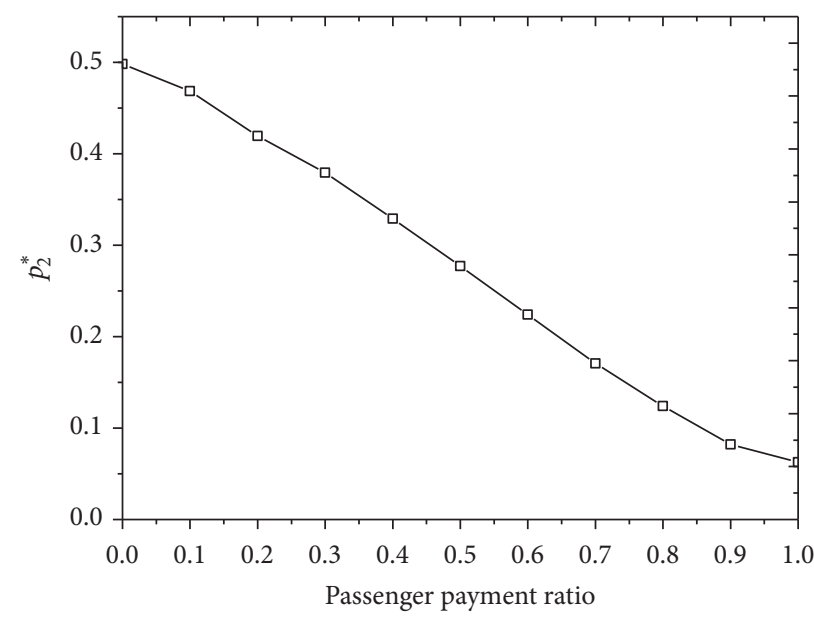

(b) Influence of payment ratio on threshold under the complaint mechanism

FIGURE 5: Influence of payment ratio on threshold.

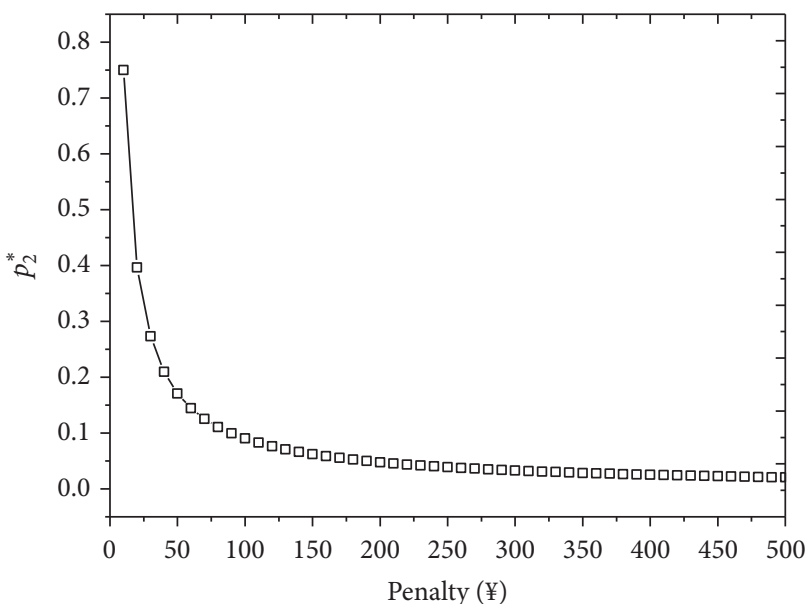

Figure 6: Influence of penalty on threshold.

threshold decreases gradually with passenger payment ratio increases. The threshold of the absence of complaint mechanism is lower obviously than that of complaint mechanism.

The influences of penalty on the threshold are shown in Figure 6. The threshold decreases gradually with penalty increases, and the rate of decline slows gradually. The threshold is about $75 \%$ when the penalty is less than $20 ¥$. The threshold remains at about $5 \%$ when the penalty is over $200 ¥$, and the continuing increase of the penalty has little effect on the threshold. There is no sense to set excessive penalty, and too low penalty can not affect drivers' strategy choice. So it is necessary to set the appropriate penalty under the complaint mechanism.

In summary, the thresholds that can ensure drivers to select taking carpooling strategy are more easily satisfied under the complaints mechanism. Complaint mechanism can reduce the rate of drivers refusing passengers and makes detour possible.

\section{Conclusions}

This paper studies the problem of taxi driver's strategy choice under the condition of carpooling detour. The model combines prospect theory and evolutionary game theory and improves traditional evolutionary game model by replacing the gain with prospect value, which makes the model more fit in with the actual psychology of human beings. Through the researches and analysis, the following conclusions are obtained:

(1) The behavior of drivers refusing passengers under the condition of carpooling detour can easily happen. The passenger complaints mechanism is an effective measure to reduce the driver refusing behavior.

(2) Whether or not drivers choose the strategy of taking carpooling detour passengers depends on the initial percentage of passengers choosing carpooling strategy or complaint strategy. Under the absence of complaint mechanism, drivers' stable strategy is taking passengers if the percentage of passengers choosing carpooling strategy is more than the threshold $p_{1}^{*}$; Under the complaint mechanism, drivers' stable strategy is taking passengers if the percentage of passengers choosing complaint strategy is more than the threshold $p_{2}^{*}$.

(3) The thresholds relate to detour distance, traffic congestion rate, payment ratio, and penalty. The thresholds increase gradually with detour distance or traffic congestion rate increases. The thresholds decrease gradually with payment ratio or penalty increases. It is an effective way to avoid driver refusing behavior to limit detour distance, ease traffic congestion, and select the appropriate payment ratio and penalty.

Therefore, the impacts of the factors, such as detour distance and traffic congestion, have to be considered in the implementation process of taxi carpooling system. It is 
necessary to provide a simple and effective way of complaints to encourage passengers complaining actively in order to control refusing behavior.

\section{Conflicts of Interest}

The authors declare that they have no conflicts of interest.

\section{Acknowledgments}

This research was funded by National Natural Science Foundation of China (61364026, 51408288, and 71661021) and Youth Science Foundation of Lanzhou Jiaotong University (2015032). The authors express their thanks to all who participated in this research for their cooperation.

\section{References}

[1] D. Zhang, T. He, Y. Liu, S. Lin, and J. A. Stankovic, "A carpooling recommendation system for taxicab services," IEEE Transactions on Emerging Topics in Computing, vol. 2, no. 3, pp. 254-266, 2014.

[2] R. Javid, A. Nejat, and M. Salari, "The environmental impacts of carpooling in the United States," in Proceedings of the Transportation, Land and Air Quality Conference, 2016.

[3] J. Jamal, A. E. Rizzoli, R. Montemanni, and D. Huber, "Tour planning and ride matching for an urban social carpooling service," in Proceedings of the 5th International Conference on Transportation and Traffic Engineering (ICTTE '16), Switzerland, July 2016.

[4] W. Shen, C. V. Lopes, and J. W. Crandall, "An online mechanism for ridesharing in autonomous mobility-on-demand systems," in Proceedings of the 25th International Joint Conference on Artificial Intelligence, pp. 475-481, New York, USA, 2016.

[5] S. Galland, L. Knapen, A.-U.-H. Yasar et al., "Multi-agent simulation of individual mobility behavior in carpooling," Transportation Research Part C: Emerging Technologies, vol. 45, pp. 83-98, 2014.

[6] S.-K. Chou, M.-K. Jiau, and S.-C. Huang, "Stochastic set-based particle swarm optimization based on local exploration for solving the carpool service problem," IEEE Transactions on Cybernetics, vol. 46, no. 8, pp. 1771-1783, 2016.

[7] K. Aissat and A. Oulamara, "Dynamic ridesharing with intermediate locations," in Proceedings of the 2014 IEEE Symposium on Computational Intelligence in Vehicles and Transportation Systems (CIVTS '14), pp. 36-42, USA, December 2014.

[8] P. Santi, G. Resta, M. Szell, S. Sobolevsky, S. H. Strogatz, and C. Ratti, "Quantifying the benefits of vehicle pooling with shareability networks," Proceedings of the National Acadamy of Sciences of the United States of America, vol. 111, no. 37, pp. 13290-13294, 2014.

[9] T. Shinde and B. Thombre, "An effective approach for solving carpool service problems using genetic algorithm approach in cloud computing," International Journal of Advance Research in Computer Science and Management Studies, vol. 3, no. 12, pp. 29-33, 2015.

[10] W. He, K. Hwang, and D. Li, "Intelligent carpool routing for urban ridesharing by mining GPS trajectories," IEEE Transactions on Intelligent Transportation Systems, vol. 15, no. 5, pp. 2286-2296, 2014.
[11] M. Nourinejad and M. J. Roorda, "Agent based model for dynamic ridesharing," Transportation Research Part C: Emerging Technologies, vol. 64, pp. 117-132, 2016.

[12] S.-C. Huang, M.-K. Jiau, and C.-H. Lin, "A genetic-algorithmbased approach to solve carpool service problems in cloud computing," IEEE Transactions on Intelligent Transportation Systems, vol. 16, no. 1, pp. 352-364, 2015.

[13] J. Xia, K. M. Curtin, W. Li, and Y. Zhao, "A new model for a carpool matching service," PLoS ONE, vol. 10, no. 6, Article ID e0129257, 2015.

[14] S.-Y. Chiou and Y.-C. Chen, "A mobile, dynamic, and privacypreserving matching system for car and taxi pools," Mathematical Problems in Engineering, vol. 2014, Article ID 579031, 10 pages, 2014.

[15] C. M. Boukhater, O. Dakroub, F. Lahoud, M. Awad, and H. Artail, "An intelligent and fair GA carpooling scheduler as a social solution for greener transportation," in Proceedings of the 2014 17th IEEE Mediterranean Electrotechnical Conference (MELECON '14), pp. 182-186, Lebanon, April 2014.

[16] Q. Xiao, R.-C. He, W. Zhang, and C.-X. Ma, "Algorithm research of taxi carpooling based on fuzzy clustering and fuzzy recognition," Journal of Transportation Systems Engineering and Information Technology, vol. 14, no. 5, pp. 119-125, 2014.

[17] G. Mallard, Bounded rationality and behavioral economics, Routledge Press, London, UK, 2015.

[18] P. van den Berg and F. J. Weissing, "Evolutionary Game Theory and Personality," in Evolutionary Perspectives on Social Psychology, Evolutionary Psychology, pp. 451-463, Springer International Publishing, Cham, 2015.

[19] M. S. Eid, I. H. El-Adaway, and K. T. Coatney, "Evolutionary stable strategy for postdisaster insurance: Game theory approach," Journal of Management in Engineering, vol. 31, no. 6, Article ID 04015005, 2015.

[20] C. Wu, Y. Pei, and J. Gao, "Evolution game model of travel mode choice in metropolitan," Discrete Dynamics in Nature and Society, vol. 2015, Article ID 638972, 11 pages, 2015.

[21] C. S. Gokhale and A. Traulsen, "Evolutionary multiplayer games," Dynamic Games and Applications, vol. 4, no. 4, pp. 468488, 2014.

[22] A. Tversky and D. Kahneman, "Advances in prospect theory: cumulative representation of uncertainty," Journal of Risk and Uncertainty, vol. 5, no. 4, pp. 297-323, 1992.

[23] J. Wang and T. Sun, "Fuzzy multiple criteria decision making method based on prospect theory," in Proceedings of the Proceeding of the International Conference on Information Management, Innovation Management and Industrial Engineering (ICIII '08), vol. 1, pp. 288-291, Taipei, Taiwan, December 2008.

[24] P. A. de Castro, A. . Barreto Teodoro, L. I. de Castro, and S. Parsons, "Expected utility or prospect theory: which better fits agent-based modeling of markets?" Journal of Computational Science, vol. 17, no. part 1, pp. 97-102, 2016.

[25] W. Zhang and R. C. He, "Dynamic route choice based on prospect theory," in Proceedings of the, vol. 138, pp. 159-167.

[26] M. Abdellaoui, H. Bleichrodt, O. L'Haridon, and D. van Dolder, "Measuring loss aversion under ambiguity: a method to make prospect theory completely observable," Journal of Risk and Uncertainty, vol. 52, no. 1, pp. 1-20, 2016.

[27] L. A. Prashanth, J. Cheng, F. Michael, M. Steve, and S. Csaba, "Cumulative prospect theory meets reinforcement learning: prediction and control," in Proceedings of the 33th International Conference on Machine Learning, pp. 2112-2121, New York, NY, USA, 2016. 


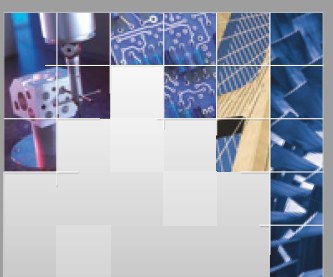

\section{Enfincering}
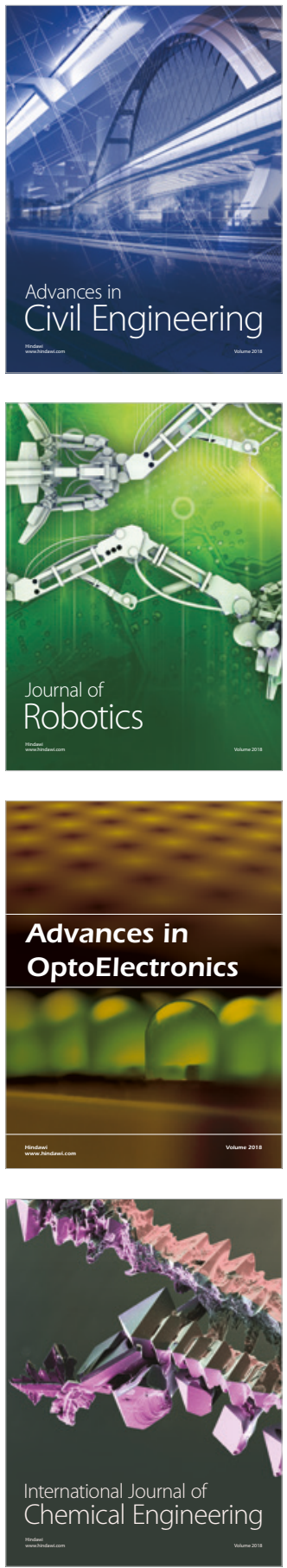

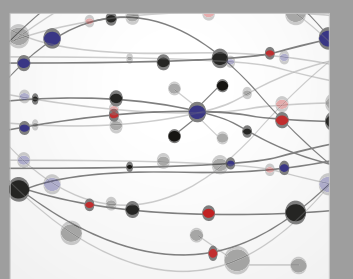

\section{Rotating \\ Machinery}

The Scientific World Journal

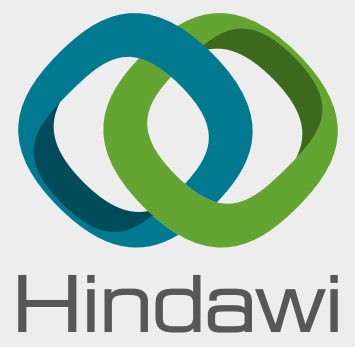

Submit your manuscripts at

www.hindawi.com
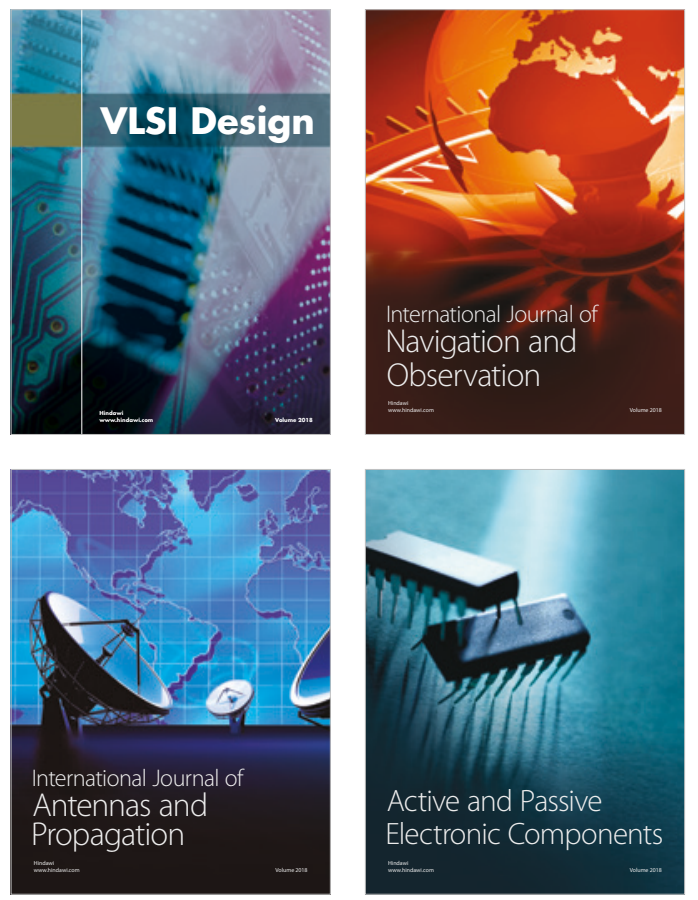
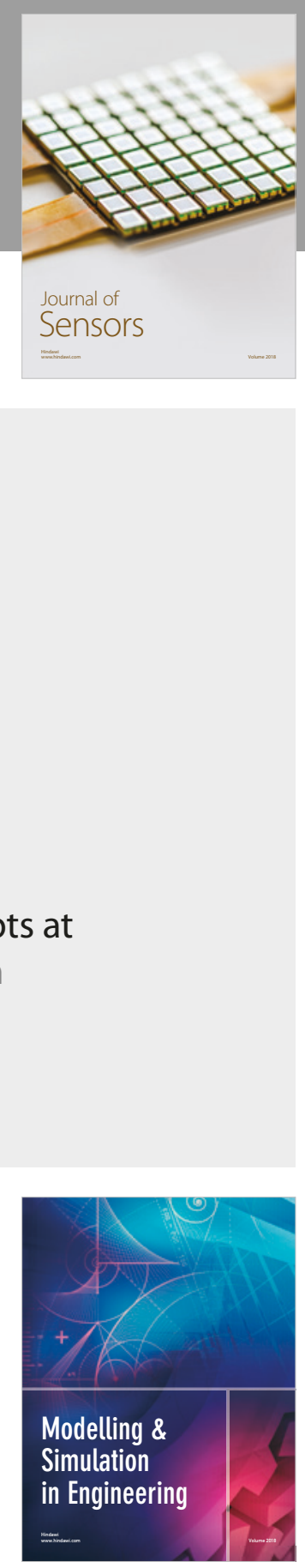

\section{Advances \\ Multimedia}
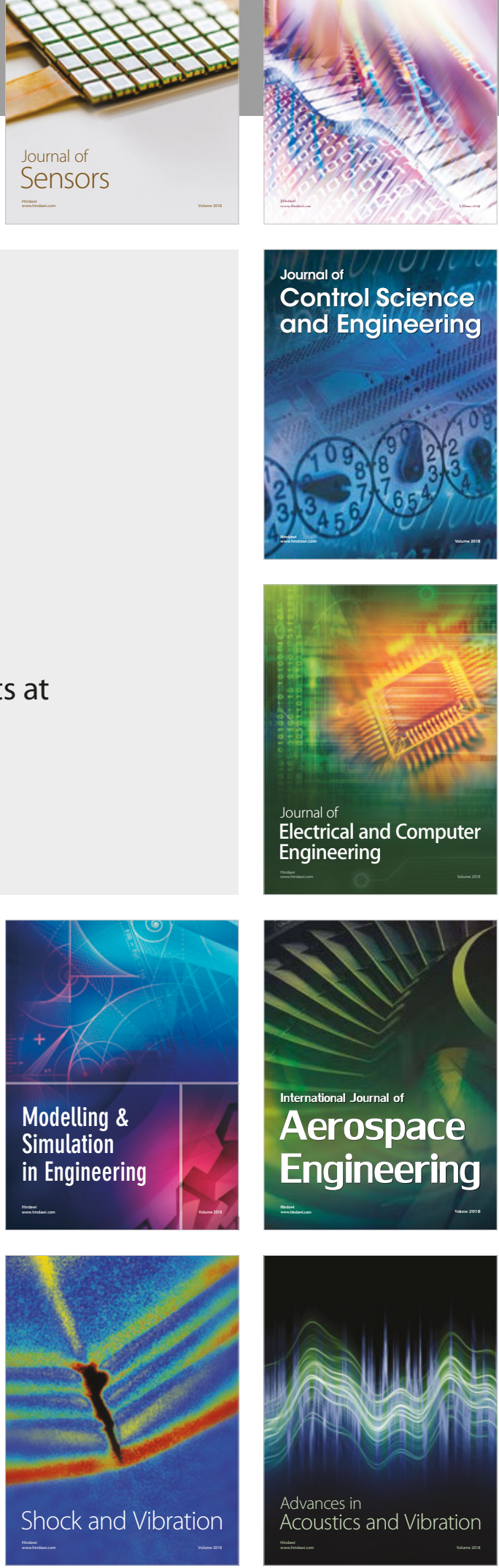